Section D:

The Next Decade 


\title{
Exoplanet transit spectro-photometry with SOFIA
}

\author{
Daniel Angerhausen ${ }^{1}$, Alfred Krabbe ${ }^{1}$ and Hans Zinnecker ${ }^{1}$ \\ ${ }^{1}$ Deutsches SOFIA Institut, Universität Stuttgart, Pfaffenwaldring 31, 70569 Stuttgart, \\ Germany \\ email: angerhausen@irs.uni-stuttgart.de
}

\begin{abstract}
We present the prospects of observing extrasolar planets with the Stratospheric Observatory for Infrared Astronomy (SOFIA). Our analysis shows that optical and near-infrared photometric and spectrophotometric follow-up observations during planetary transits and eclipses will be feasible with SOFIA's instrumentation, especially with the HIPO-FLITECAM optical/NIR instruments. SOFIA has unique advantages in comparison to ground- and spacebased observatories in this field of research which will be outlined.
\end{abstract}

Keywords. planetary systems, telescopes

\section{Introduction}

The Stratospheric Observatory for Infrared Astronomy (SOFIA) is a NASA 80:20 partnership with the German Space Agency (DLR) to develop a Boeing 747SP airliner fitted with a 2.7-meter reflecting telescope, making it the largest airborne observatory in the world (Gehrz et al. 2009). SOFIA has successfully had its first light observation in May 2010. Its first generation of science instruments consists of 7 imagers and spectrographs spanning the entire wavelength range from the optical to the far infrared $(0.3-200 \mu \mathrm{m})$. As warm Spitzer winds down and the future role of HST's infrared capabilities is uncertain, ground-based and SOFIA observations will play a dominant role for exoplanet atmosphere observations in the next decade, before JWST starts operating (see fig. 1).

\section{Advantages of SOFIA for exoplanet observations}

Even the very close-in extrasolar planets, with distances to their host star of only a few stellar radii, are not much hotter than $T \simeq 2000 K$. Therefore the equivalent blackbody emission always peaks in the infrared. Furthermore many important atmospherical properties such as the chemical constituents or the T-P-profiles of planets can be analyzed with IR spectra, observed during transits or eclipses of the planet (e.g. Swain et al. 2009). From this perspective, SOFIA operates in the optimal wavelength regime for exoplanet observations.

For short-period close-in transiting planets with transits and eclipses occurring every 25 days, the optimal observing schedules for ground-based transit observations are reduced to only few nights per year for a given observing site as the event is best observed close to target culmination and local midnight. The HST, on the other hand, is able to observe transits at many more opportunities but is limited to series of 96 minute on/off-target batches due to its near-earth orbit. This presents a substantial hurdle - in particular for transiting planets with very long orbits (such as HD80606b) and therefore transit durations of more than 6 hours. The analysis of potential flight schedules shows that the mobile platform SOFIA will be able to take off close to the optimal geographic location 


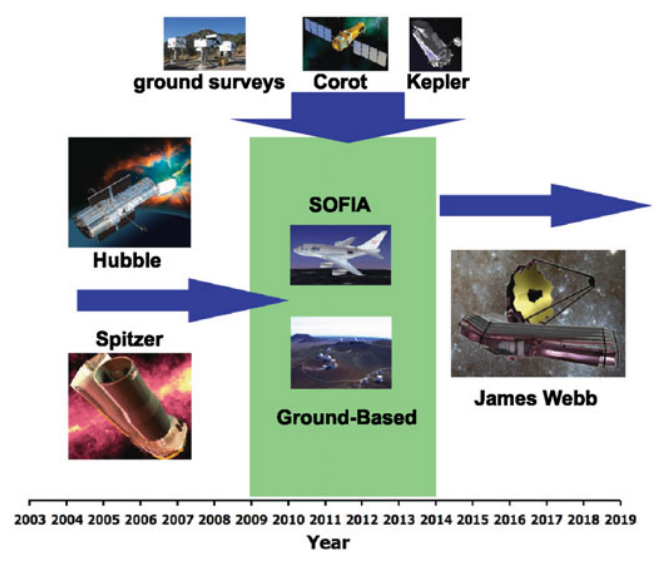

Figure 1. Diagram to explain the perspectives of transit spectro-photometry in the next decade. In the past few years Hubble and Spitzer (left) have been successfully used for spectroscopic characterization of extrasolar planets. As warm Spitzer winds down and the future role of HST's infrared capabilities is uncertain, space-based opportunities are limited until JWST starts operating. For the next years ground-based surveys, CoRoT and Kepler (top) are going to deliver a large number of new targets with interesting opportunities for characterization. The airborne observatory SOFIA will help to follow-up these planets and as testbed for future JWST observations.

for each of those events. The airborne observatory will be able to observe the complete event continuously and with a very stable setup (telescope elevation, airmass etc.) during observing flights of up to 10 hours duration.

The variability of earth's atmospheric transmission as well as the temporal variability of its constituents is the most crucial challenge in ground-based transit observations, in particular when it comes to the spectroscopic analysis of molecular features in the exoplanet's atmosphere that are also present as telluric trace gases. Again, SOFIA will also deal very favorably with these effects since it will be able to fly high enough (12-14 $\mathrm{km})$ to be independent of near surface processes affecting in particular the water and methane lines. The SOFIA telescope is operating at much lower temperatures $(240 \mathrm{~K})$ than ground-based telescopes. Therefore thermal background contributions, that are the dominant noise source for transit observations at wavelengths longer than 3 micron, will be significantly reduced (Angerhausen et al. 2010).

\section{Summary}

We demonstrate that SOFIA has a specific and unique phase space for exoplanet research in the next decade. SOFIA operates in the right wavelength regime, above most of the perturbing variation of atmospheric trace gases and can observe rare transient events under optimized conditions. SOFIA will instantaneously be a competitive observatory in the field of state of the art exoplanet astronomy and some science topics are even exclusively observable with SOFIA.

\section{References}

Angerhausen, D., Krabbe, A., \& Iserlohe, C. 2010, PASP, 122, 1020

Gehrz, R. D., Becklin, E. E., de Pater, I., Lester, D. F., Roellig, T. L., \& Woodward, C. E. 2009, AdSR, 44, 413

Swain, M. R., et al. 2009, ApJ, 704, 1616 